\title{
Location Prediction of Mobility Management Using Soft Computing Techniques in Cellular Network
}

\author{
Smita Parija ${ }^{a *}$, Santosh Kumar Nanda ${ }^{b}$, Prasanna Kumar Sahu ${ }^{c}$, Sudhansu Sekhar Singh ${ }^{d}$ \\ ${ }^{\mathrm{a}, \mathrm{c},}$, Department of Electrical engineering, National Institute of Technology, Rourkela-759028 \\ a smita.parija@gmail.com, ${ }^{\mathrm{c}}$ pksahu@nitrkl.ac.in \\ ${ }^{\mathrm{b}}$ Centre of Research, Development and Consultancy, Eastern Academy of Science and \\ Technology, Bhubaneswar, Odisha, India - 754001, \\ bsknanda@eastodissa.ac.in \\ ${ }^{\mathrm{d}}$ Department of Electronics Engineering, KIIT University, BBSR \\ dssinghfet@kiit.ac.in
}

\begin{abstract}
This work describes the neural network technique to solve location management problem. A multilayer neural model is designed to predict the future prediction of the subscriber based on the past predicted information of the subscriber. In this research work, a prediction based location management scheme is proposed for locating a mobile terminal in a communication without losing quality maintains a good response. There are various methods of location management schemes for prediction of the mobile user. Based on individual characteristic of the user, prediction based location management can be implemented. This work is purely analytical which need the past movement of the subscriber and compared with the simulated one. The movement of the mobile target is considered as regular and uniform. An artificial neural network model is used for mobility management to reduce the total cost. Single or multiple mobile targets can be predicted. Among all the neural techniques multilayer perceptron is used for this work. The records are collected from the past movement and are used to train the network for the future prediction. The analytical result of the prediction method is found to be satisfactory.
\end{abstract}

Index Terms - Cellular Network, Mobility Management,Neural Network, Multi layer perceptron

\section{INTRODUCTION}

Location management is one of the major issues in mobile networks to provide an efficient and low-cost services In cellular communication network, when a call arrives to a subscriber it is required to collect the information about the location of the mobile host. In a cellular communication network the user movement are normally preplanned and the network reserves the information of the entire subscriber in its system data base. The mobile terminal is free to move within the entire environment. There are several issues in mobile cellular environment which is usually conceived as a collection of cells where each served by base station located at its mobile switching centre (MSC). The number of cells are related to the MSC which acts as a gateway of the cellular network. The problems in this area are clearly mentioned i.e. cost factor increases when the mobility of the user in a particular coverage increases. This paper presents an issue based on location management, which is an important criteria in mobile networking that roaming users with mobile station to use their services through radio network when they are jumping to a new service area. Mobile systems use the location update concept and location update procedure in order to keep the track of the user location. More specifically cells are grouped into location areas. Periodically a roaming mobile user that is not involved in a call obtains the identifier of the location area to which the nearest base station belongs. When there is a change in the location area identifier is sensed, the location update procedure is raised. By means of this procedure the identifier of the new location area is stored in the system data base.

Similarly when the call has to route from the network to a mobile host, the current cell of the mobile terminal should be determined. Mobile systems in order to get the information apply the paging area concept and paging area procedure. In the cells of the location area a message is broadcast which contents indicate the mobile terminal has an incoming call. When mobile terminal responds from a certain cell, its exact position as well as its availability to accept the call are determined.The location update and paging procedure impose a significant load on the system that is also transferred over the radio interface which is a scarce resource. Hence for the acceptable quality of services at low cost, the technique which controls the signaling load and especially, the impact on the radio interface are required. In cellular networks, several attempts have been made to reduce traffic load [1]. One of the methods is to use mobile terminal location movement information and their traffic properties to predict the future location of a mobile terminal. If the next location of mobile terminal is known in terms of distance/direction in advance, then there is no explicit 
location updating is required and paging throughout the geographical area can be avoided, thereby reducing system overload.

Location update scheme are often partitioned into static and dynamic. A location update is static if a predetermined set of cell location updating occur on either same intervals or upon every cell change. On the other hand dynamic location management is an advanced concept where the parameters of location management can be modified to be best fitted for individual users and conditions [2]. However, dynamic location management concepts are excessively theoretical and complex, and a tuff task to implement on a large scale. Location management is a very important issue as it includes location updating \& paging. The process of location update enables the network to find the position of the mobile device \& paging is used to poll a set of cell to determine the precise location of a device to route an incoming call to maintain the quality of service [3].

In this paper, a neural network scheme using back propagation technique is proposed for location prediction of mobile host. The future position of a mobile terminal is predicted by this algorithm based on the previous record of its movement pattern. The data collected is uniform and regular. The network is trained with the data obtained from the past history of movement pattern of a MT for making predictions for future movement [4-5]. This paper is organized as follows. In Section II, Location update technique is illustrated. Neural model is developed in Section III .Analysis of the model and related work is explained in this section and finally conclusion and references are given in Section IV and V.

\section{II.PROPOSED TECHNIQUE FOR LOCATION UPDATE IN MOBILITY MANAGEMENT}

The proposed method for mobile movement prediction is based on the MT's history of movement patterns, which has been recorded for certain time duration. A multi-layer artificial neural network is used to process the movement pattern of mobile terminal for accurate prediction of mobile movements.

Movement pattern $\left(\mathrm{P}_{\mathrm{n}}\right)$ is the history of movement of a mobile terminal recorded for a period of time interval $T_{n}$, where $\mathrm{n}$ is the number of successive time intervals at which the mobile terminal movements are recorded. The successive time interval can be minutes, hours, days, etc. The movement pattern $\mathrm{P}_{\mathrm{n}}$ which is used for the training purpose can be represented by a numeric data in terms of distance and direction at the successive time interval, $t_{1}$, $t_{2}, \ldots t_{n}$.

Let the movement pattern $\mathrm{P}_{\mathrm{n}}=\left\{\mathrm{p}_{1}, \mathrm{p}_{2}, \ldots, \mathrm{p}_{\mathrm{n}}\right\}$ be recorded for an $\mathrm{MT}$, where $\mathrm{Pj}$ indicates the movement of a mobile host during time $t_{j}$, and we define the movement in terms of distance and direction travelled by the MT during the time interval $t_{j}$. Then $P_{j}$ is represented by a pair $\left(\mathrm{ds}_{\mathrm{j}}, \mathrm{dr}_{\mathrm{j}}\right)$ where $\mathrm{ds}_{\mathrm{j}}$ is the distance travelled by a mobile host at $j^{\text {th }}$ time interval which may be number of cells, kilometres, meters etc. and $\mathrm{dr}_{\mathrm{j}}$ is the possible direction in which a mobile host moves at $\mathrm{j}^{\text {th }}$ time interval[4].
For example, if a mobile movement pattern is recorded for two time intervals $(n=2)$ with direction of movements South and West and the distance travelled is 4 and 5 units, then the movement pattern is, $\mathrm{P}_{2}=\left\{\mathrm{p}_{1}, \mathrm{p}_{2}\right\}=\left\{\left(\mathrm{ds}_{1}, \mathrm{dr}_{1}\right)\right.$, $\left.\left(\mathrm{ds}_{2}, \mathrm{dr}_{2}\right)\right\}=\{(4$, South $),(5$, West $)\}$. Training data set is the set of sub patterns obtained from the movement pattern $\mathrm{p}_{\mathrm{n}}$ by dividing it into $\mathrm{u}$ sub patterns, where $\mathrm{u}+1$ is the size of each sub pattern $(u<<n)$. The sub pattern is a training data pair with mobile the sub pattern is a training data pair with mobile movements for $u$ time intervals as input and the movement for the next time interval as a desired output. For example, the first training sub pattern is $\mathrm{p}_{1}, \mathrm{p}_{2}, . . \mathrm{p}_{\mathrm{u}}$ as input and $\mathrm{p}_{\mathrm{u}+1}$ as the desired output $[5,6]$.

The parameter $\mathrm{u}$ is the prediction order or time window, which is normally selected based on the movement characteristics of a mobile terminal and the size of the recorded movement pattern .

In our work, we have considered two mobiles MT1 and MT2 with regular and random movement patterns respectively. Their movement patterns are given in terms of distance in meter the mobile has crossed and the direction of movement. The training data sets for MT1 and MT2 are given in Table 1 and 2 respectively.

For example, if a mobile movement pattern is $\operatorname{dis}_{1}$ and $\operatorname{dir}_{1}=$ MT distance and direction during the first time interval; $\operatorname{dis}_{2}$ and $\operatorname{dir}_{2}=$ MT distance and direction during the second time interval; and so on and $\operatorname{dis}_{4}$ and $\operatorname{dir}_{4}=$ MT distance and direction observed at fourth time interval, i.e. desired output for the given input training data[7].

The directions are represented as follow -

North -1 ,East -2 ,South -3 , West -4 , North-East - 12, South-East - 32, South-West - 34, North-West - 14

Table I: Training data set for MT1

\begin{tabular}{|c|c|c|c|}
\hline $\mathrm{ds} 1, \mathrm{dr} 1$ & $\mathrm{ds} 2, \mathrm{dr} 2$ & $\mathrm{ds} 3, \mathrm{dr} 3$ & Output:ds4,dr4 \\
\hline$(2,14)$ & $(2,4)$ & $(2,14)$ & $(2,4)$ \\
\hline$(2,4)$ & $(2,14)$ & $(2,4)$ & $(2,14)$ \\
\hline$(2,14)$ & $(2,4)$ & $(2,14)$ & $(2,4)$ \\
\hline$(2,4)$ & $(2,14)$ & $(2,4)$ & $(2,14)$ \\
\hline$(2,14)$ & $(2,4)$ & $(2,14)$ & $(?, ?)$ \\
\hline
\end{tabular}

Table II: Training data set for MT2

\begin{tabular}{|c|c|c|c|c|}
\hline $\mathrm{ds} 1, \mathrm{dr} 1$ & $\mathrm{ds} 2, \mathrm{dr} 2$ & $\mathrm{ds} 3, \mathrm{dr} 3$ & $\mathrm{ds} 4, \mathrm{dr} 4$ & $\begin{array}{c}\text { Output: } \\
\mathrm{ds} 5, \mathrm{dr} 5\end{array}$ \\
\hline$(2,4)$ & $(2,34)$ & $(3,4)$ & $(2,3)$ & $(2,4)$ \\
\hline$(2,34)$ & $(3,4)$ & $(2,3)$ & $(2,4)$ & $(2,12)$ \\
\hline$(3,4)$ & $(2,3)$ & $(2,4)$ & $(2,12)$ & $(2,4)$ \\
\hline$(2,3)$ & $(2,4)$ & $(2,12)$ & $(2,4)$ & $(3,3)$ \\
\hline$(2,4)$ & $(2,12)$ & $(2,4)$ & $(3,3)$ & $(2,4)$ \\
\hline$(2,12)$ & $(2,4)$ & $(3,3)$ & $(2,4)$ & $(2,32)$ \\
\hline$(2,4)$ & $(3,3)$ & $(2,4)$ & $(2,32)$ & $(2,2)$ \\
\hline$(3,3)$ & $(2,4)$ & $(2,32)$ & $(2,2)$ & $(2,1)$ \\
\hline$(2,4)$ & $(2,32)$ & $(2,2)$ & $(2,1)$ & $(3,2)$ \\
\hline$(2,32)$ & $(2,2)$ & $(2,1)$ & $(3,2)$ & $(2,34)$ \\
\hline$(2,2)$ & $(2,1)$ & $(3,2)$ & $(2,34)$ & $(3,2)$ \\
\hline$(2,1)$ & $(3,2)$ & $(2,34)$ & $(3,2)$ & $(?, ?)$ \\
\hline
\end{tabular}


By observing the directional changes in the movement pattern, suitable prediction order $(\mathrm{k})$ is considered and the corresponding sub patterns are obtained. Here, the size of sub pattern is kept to be 6 . The neural network is rained with all the sub patterns to predict the movement $(?, ?)$ of Table 1 and 2 . The same data set are then used to predict multiple moves by updating the data set given in Table 1 and 2.

\section{A NEURAL NETWORK MODEL FOR LOCATION UPDATE}

A mobile cellular environment is considered with rectangular array of cells in which the mobile stations can move freely. The possible direction which a mobile terminal can move is considered from the direction set $\mathrm{D}=\{$ North, East, South, west, North East, South east, south West, North West $\}=\{1,2,3,4,12,32,34$, $14\}$. The distance is recorded in terms of the number of cells travelled by a mobile station at each time interval. This distance is taken as the training inputs for our neural network model as shown in Fig.1 [8]. A three layer neural network with 8 hidden neurons was used to develop the model for mobile movement prediction. The training sets are formed from the data for direction of the mobile host in a particular time interval and the corresponding direction of the mobile host in next time interval is taken for output of the training pattern. A gradient decent back propagation algorithm has been used to optimize the weight vectors of the neural network model. A sigmoid function is used as activation function and eleven different data sets were used to train the neural network for optimized movement pattern. The predicted distance/direction is determined by using the trained neural network model after proper learning [9]. The testing of the neural network model has been done with some arbitrary numeric data in terms of distance/direction which have not been used in the training process. Multiple prediction results can be obtained from the trained neural network model with successive data sets. In the location prediction, with the help of the neural networks a relation between the past and the future values of the movement pattern is derived which helps in predicting the future location of the mobile terminal for location management [10].

The single movement prediction result for MT1 is found to be $(3,4)$ i.e. it moves three meters in West direction. Similarly, the predicted movement of MT2 is found to be $(4,3)$ i.e. it will move 4 meters in South directions. Then the data of Table 1 and 2 are updated using this predicted movement and the multiple predictions is carried out using the same model as shown in Table 3 and 4 respectively. The predicted output is shown by bold letters [11]. Based on the available historical data several techniques have-been proposed for future location prediction in cellular network.

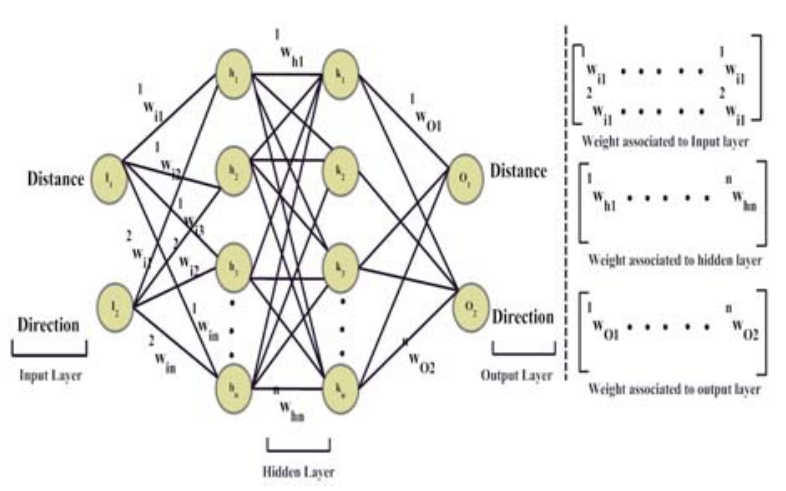

Figure:1 Development neural network

Table III: Training data set for MT1 and Prediction results

\begin{tabular}{|l|l|l|l|}
\hline ds1, dr1 & ds2, dr2 & ds3, dr3 & $\begin{array}{l}\text { Output :ds4, } \\
\text { dr4 }\end{array}$ \\
\hline$(2,14)$ & $(2,4)$ & $(2,14)$ & $(2,4)$ \\
\hline$(2,4)$ & $(2,14)$ & $(2,4)$ & $(2,14)$ \\
\hline$(2,14)$ & $(2,4)$ & $(2,14)$ & $(2,4)$ \\
\hline$(2,4)$ & $(2,14)$ & $(2,4)$ & $(2,14)$ \\
\hline$(2,14)$ & $(2,4)$ & $(2,14)$ & $(2,4)$ \\
\hline$(2,4)$ & $(2,14)$ & $(2,4)$ & $(2,14)$ \\
\hline$(2,14)$ & $(2,4)$ & $(2,14)$ & $(2,4)$ \\
\hline$(2,4)$ & $(2,14)$ & $(2,4)$ & $(2,14)$ \\
\hline$(2,14)$ & $(2,4)$ & $(2,14)$ & $(2,4)$ \\
\hline
\end{tabular}

Table IV: Training data set for MT2 and Prediction results

\begin{tabular}{|c|c|c|c|c|}
\hline ds1, dr1 & ds2, dr2 & ds3, dr3 & ds4, dr4 & $\begin{array}{c}\text { Output : } \\
\text { ds5,dr5 }\end{array}$ \\
\hline$(2,4)$ & $(2,34)$ & $(3,4)$ & $(2,3)$ & $(2,4)$ \\
\hline$(2,34)$ & $(3,4)$ & $(2,3)$ & $(2,4)$ & $(2,12)$ \\
\hline$(3,4)$ & $(2,3)$ & $(2,4)$ & $(2,12)$ & $(2,4)$ \\
\hline$(2,3)$ & $(2,4)$ & $(2,12)$ & $(2,4)$ & $(3,3)$ \\
\hline$(2,4)$ & $(2,12)$ & $(2,4)$ & $(3,3)$ & $(2,4)$ \\
\hline$(2,12)$ & $(2,4)$ & $(3,3)$ & $(2,4)$ & $(2,32)$ \\
\hline$(2,4)$ & $(3,3)$ & $(2,4)$ & $(2,32)$ & $(2,2)$ \\
\hline$(3,3)$ & $(2,4)$ & $(2,32)$ & $(2,2)$ & $(2,1)$ \\
\hline$(2,4)$ & $(2,32)$ & $(2,2)$ & $(2,1)$ & $(2,1)$ \\
\hline$(2,32)$ & $(2,2)$ & $(2,1)$ & $(3,2)$ & $(2,34)$ \\
\hline$(2,2)$ & $(2,1)$ & $(3,2)$ & $(2,34)$ & $(3,2)$ \\
\hline$(2,1)$ & $(3,2)$ & $(2,34)$ & $(3,2)$ & $(4,2)$ \\
\hline$(3,2)$ & $(2,34)$ & $(3,2)$ & $(4,2)$ & $(2,23)$ \\
\hline$(2,34)$ & $(3,2)$ & $(4,2)$ & $(2,23)$ & $(2,3)$ \\
\hline$(3,2)$ & $(4,2)$ & $(2,23)$ & $(2,3)$ & $(2,3)$ \\
\hline$(4,2)$ & $(2,23)$ & $(2,3)$ & $(2,3)$ & $(2,3)$ \\
\hline
\end{tabular}

\section{COMPARISION OF PROPOSED ANALYTICAL METHOD WITH SIMULATED METHOD}


The multilayer neural network frame for mobile movement prediction is based on the back propagation learning algorithm [12]. Here, the neural networks are trained with respect to previous movement pattern for learning optimized functions for predictions. The task of the neural networks in this application is to capture the unknown relation between the past and the future values of the movement pattern. This helps in predicting the future location of a mobile host for location management [13].

B. Selection of neurons for multilayer neural network model

The most important factor to be considered is to develop an artificial neural network model of appropriate size for capturing the underlying movement patterns in the training data. The selection of number of input and hidden layer neurons will largely fix the size of the multilayer neural network model. Multilayer feed forward neural network learned by back propagation based on supervised learning method. Here the non linear neurons are arranged in a successive layer and the information flow uni-directionally from input layer to output layer through hidden layer. The neural network model we consider for prediction consists of three layers: input, hidden and output. The number of input neurons is an important parameter since it corresponds to the length of the sub patterns used to discover the underlying features in a given movement data. The multilayer perceptron we have considered here has the following characteristics [14].

(i) It has two numbers of inputs in term of distance and direction.

(ii) It has one number of hidden layer consist of neurons which only receive the information and produce the internal output to other processing unit.

(iii) Uses linear combination function in the hidden and output layer.

(iv) Uses generally sigmoid activation function in the hidden layer.

(v) It has two numbers of neurons in the output layer as movement parameters are distance and direction.

Training procedure: The training is done by using back propagation in two ways. The forward pass is used to evaluate the output of the neural network for the given input in the existing weights. In the reverse pass, the difference in the neural network output with the desired output is compared and fed back to the neural network as an error to change the weights of the neural network

In ANN from the name multi means more than one layers of neurons and in layers these neurons are arranged in groups. The different layers are input layer hidden layer and output layer. MLP is feed-forward network with one or more numbers of nodes present in the hidden layers [15-16].

\section{Application of Multi Layer-Perceptron based solution} to Location prediction:
Stepwise algorithm of MLP system is represented as following manner:

Step 1: Select the total number of layers $m$, the number $\mathrm{n}_{\mathrm{i}}(\mathrm{i}=1,2, \ldots, \mathrm{m}-1)$ of the neurons in each hidden layer and an error tolerance parameter 0 .

Step 2: Randomly select the initial values of the weight vectors $w^{m} i, j$, for $\mathrm{i}=1,2, \ldots$ ni and $\mathrm{m}=2$ (number of layers).

Step 3(Initialization): All the weights $w^{m} i, j$ were initialized to random number and given as $w^{m} i, j(0)$

$$
w^{m} i, j \longleftarrow w^{m} i, j(0)
$$

Step 4 (Calculation of the neural outputs):

$$
\left\{\begin{array}{l}
a^{m}{ }_{i, j}=\left(w_{i, j}^{1}\right) \times X_{k} \\
y_{j}=\left(w^{2}{ }_{i, j}\right) \times a^{m}{ }_{j}
\end{array}\right.
$$

Step 5 (Calculation of the output error):

The error was calculated as

$$
e j=d j-y j
$$

It may be seen that the network produces a scalar output.

Step 6 (Calculation of the derivative of network output of each layer):

For hidden layer $(m=1)$

The derivative of activation function of hidden layer can be represented as

$$
\begin{aligned}
\dot{f}^{1}\left(n^{1}\right)= & \frac{d}{d n}\left(\frac{1}{1+\exp ^{-n}}\right)=\left(1-\frac{1}{1+\exp ^{-n}}\right) \\
& \times\left(\frac{1}{1+\exp ^{-n}}\right)=\left(1-a^{m}{ }_{i, j}\right)\left(a^{m}{ }_{i, j}\right)
\end{aligned}
$$

For output layer $(m=2)$

The derivative of activation function of output layer can be represented as

$$
\dot{f}^{2}\left(n^{2}\right)=\frac{d}{d n}(n)=1
$$

Step 7 (Back-propagation of error by sensitivities at each layer):

Back-propagation of error by sensitivities at each layer was calculated as follows:

For output layer $(m=2)$

$$
O_{j}=\tanh \left(\sum_{i=1}^{N} w_{i} \times X_{i}\right)
$$

For hidden layer $(m=1)$ 


$$
\begin{aligned}
& s_{j}^{1}=\dot{F}^{1}\left(n^{2}\right)\left(w^{2}{ }_{i, j}\right)^{T} s_{j}{ }_{j}= \\
& {\left[\begin{array}{cccc}
\left(1-a_{i, j}^{1}\right)\left(a_{i, j}^{1}\right) & 0 & \cdots & 0 \\
0 & \left(1-a_{i, j}^{2}\right)\left(a_{i, j}^{2}\right) & \cdots & 0 \\
\vdots & \vdots & \cdots & \vdots \\
0 & 0 & \cdots & \left(1-a_{n_{i}, j}^{1}\right)\left(a_{n_{i}, j}^{1}\right)
\end{array}\right]} \\
& \times\left(w_{i, j}^{2}\right)^{T} s_{j}^{2} \\
& s_{j}^{2}=-2 \dot{F}^{2}\left(n^{2}\right)\left(d_{j}-y_{j}\right)=-2 \dot{f}^{2}\left(n^{2}\right) \times\left(e_{j}\right)
\end{aligned}
$$

\section{Step 8 (Updating the weight vectors) :}

The weight matrices are updated next using the following relationship

$$
\begin{aligned}
& w_{i, j}^{2}(\text { new })=w_{i, j}^{2}(\text { old })+\eta s_{j}^{2}\left(a_{i, j}^{1}\right)^{T} \\
& w_{i, j}^{1}(\text { new })=w_{i, j}^{1}(\text { old })+\eta s_{j}^{1}
\end{aligned}
$$

\section{Step 9:}

If error $\leq \varepsilon(0.01)$ then go to Step 10 , otherwise go to Step 3.

\section{Step 10:}

After the learning is completed, the weights were fixed and the network can be used for testing.

\section{RESULT AND DISCUSSION}

To solve the proposed method, here ANN methodologies (Multi Layer Perceptorn) are adopted. This entire simulation is done using the MATLAB simulation environment. This proposed system was simulated with the help of Pentium Core due Processor 3.2 GHz CPU, 2 GB based RAM and $300 \mathrm{~GB}$ storage capacity based Personal Computer. Using the mathematical relationship, the model (MLP) was applied to predict the location management of the cellular network. Figure. 2 represents the performance of the proposed method as representing the MSE plot.

Table v: description of the overall accuracy of training and testing for the location prediction.

\begin{tabular}{|c|c|}
\hline Description & Results \\
\hline Overall training accuracy & $85 \%$ \\
\hline Overall testing accuracy & $84 \%$ \\
\hline
\end{tabular}

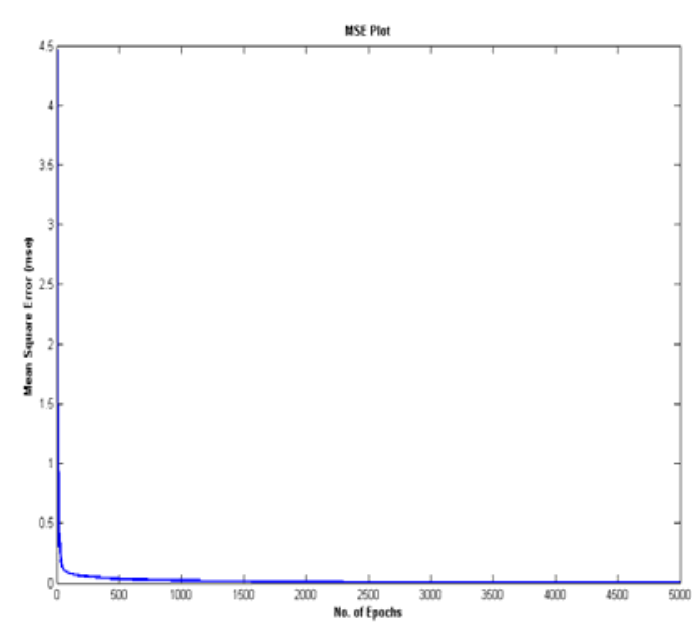

Figure 2: Mean Square Error of the proposed model

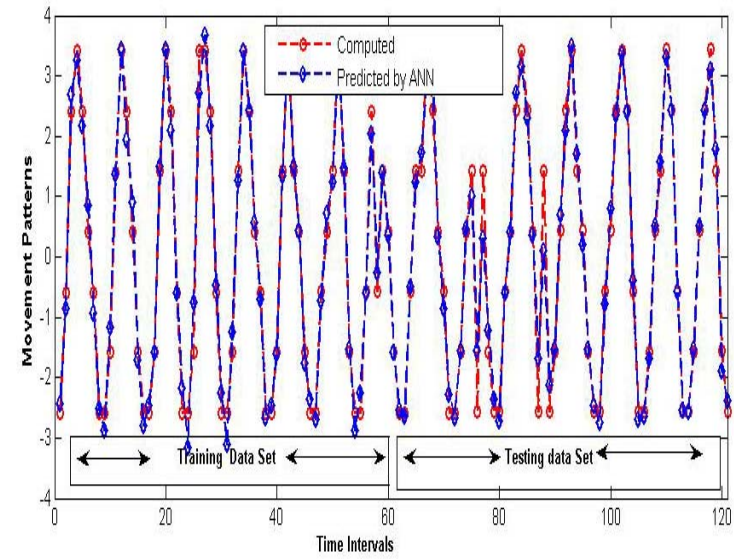

Figure 3: Performance analysis of the proposed model

The figure 3 represents prediction result of the proposed model. From the simulation result it was found that MLP based location prediction system give appropriate result with the real output. Including above results, The Table $\mathrm{V}$ describes the overall training and testing accuracy. The results shown in the Table are the overall accuracy outcome for both training and testing accuracy obtained from the trained neural network using back-propagation classifier. The overall training and testing accuracy was $85 \%$ and $84 \%$ respectively.

\section{CONCLUSION AND FUTURE WORK}

MLP-BP is a simple and able to resolve nonlinearly separable problems, a number of neurons are connected in layers to build a multilayer perceptron where each of them is used to identify small linearly separable sections of the inputs and is used to get the final output in the network. This technique is used for predict the single or multiple mobile terminals which are moving in the cellular network. This analytical prediction result is used for location update information in the cellular network Here the analytical data compared with the simulated result .It will be good research work in the direction of 
Fuzzy and Neuro-Fuzzy which do not have any well defined pattern. This paper is worked for the subscriber which shows the uniform and regular movement pattern but this work can be extended for random data.

\section{REFERENCES}

[1] I.F. Akyildiz, W. Wang, The predictive user mobility profile framework for wireless multimedia networks, IEEE/ACM Transactions on Networking 12 (6) ,pp. 1021-1035,2004 doi>10.1109/TNET.2004.838604

[2] Sunan N Huang., K. K. Tan and T. H. Lee, "Further results on adaptive control for a class of nonlinear systems using neural networks," IEEE Trans. on Neural Networks, vol. 14, no. 3, pp. 719-722, 2003. D O .I: $10.1109 /$ TNN.2003.811712

[3] Naira Hovakimyan, Flavio Nardi, Anthony Calise, and Nakwan Kim, "Adaptive output feedback control of uncertain nonlinear systems using single-hiddenlayer neural networks," IEEE Trans. on Neural Networks, vol. 13, no. 6, pp. 1420-1431, 2002.

D O I: $10.1109 /$ TNN.2002.804289

[4] L.P. Araujo, J.R.B. de Marca, A comparative analysis of paging and location update strategies for PCS networks, in: IEEE ICC_98, June, 1998, pp. 13901394. D O I: $10.1109 / \mathrm{ICC} .1998 .683055$

[5] A. Bhattacharya, S.K. Das, and LeZi-update: an information theoretic approach to track mobile users in PCS networks, in: ACM/IEEE MobiCom_99, August, 1999, pp. 1-12. doi $>10.1145 / 313451.313457$

[6] Y. Fang, I. Chlamtac, Y.-B. Lin, Portable movement modelling for PCS networks, IEEE Transactions on Vehicular Technology 49 (4) (2000).

[7] J. Korhonen, Introduction to $3 \mathrm{G}$ Mobile Communications, Artech House, Boston, MA, 2003.

[8] T. Liu, P. Bahl, I. Chlamtac, Mobility modelling, location tracking, and trajectory prediction in wireless ATM networks, IEEE Journal on Selected Areas in Communications (JSAC) 16 (6) (1998) 389-400. D O. I. 10.1109/49.709453

[9] S. Mishra, O.K. Tonguz, New metric for analyzing multistep paging schemes in mobile networks, in: IEEE VTC 2001, vol. 4, May, 2001, pp. 2590-2594. D.O I: $10.1109 /$ VETECS.2001.944069

[10] A. Misra, A. Roy, S.K. Das, An information theoretic framework for optimal location tracking in multi-system 4G wireless networks, in: Proceedings of IEEE INFOCOM 2004, June, 2004.

[11] Z. Naor, H. Levy. Minimizing the wireless cost of tracking mobile users: an adaptive threshold scheme, in: IEEE INFOCOM_98, March, 1998, pp. 720-727. D O.I: 10.1109/INFCOM.1998.665094

[12] A.-C. Pang, Y.-B. Lin, H.-M. Tsai, P. Agarwal, Serving radio network controller relocation for umts all-ip networks, IEEE Journal on Selected Areas in Communications 22 (4) (2004) 617-629. D O I: 10.1109/JSAC.2004.825962

[13] Guez Allon, James L. Ellbert and Moshe Kam, "Neural networks architecture for control," IEEE
Control System magazine, pp. 22-25, 1998. DOI:10.1109/37.1869

[14] Rivals Isabelle and Léon Personnaz, "Nonlinear internal model control using neural networks: Application to processes with delay and design issues," IEEE Trans. on Neural Networks, vol. 11, no.1, pp. 80-90, 2000. DOI:10.1109/72.822512

[15] $\mathrm{Yu}$ Wen and Li Xiaoou, "Some new results on system identification with dynamic neural networks," IEEE Transactions on Neural Networks, vol.12, no. 2, pp. 412-417, 2001. DOI:10.1109/72.914535

[16] Santosh Kumar Nanda, Debi Prasad Tripathy, and Sarat Kumar Patra. "A soft computing system for opencast mining machineries noise prediction." Noise Control Engineering Journal, Vol. 59, no. 5, pp 432446, 2011. DOI: http://dx.doi.org/10.3397/1.3614042

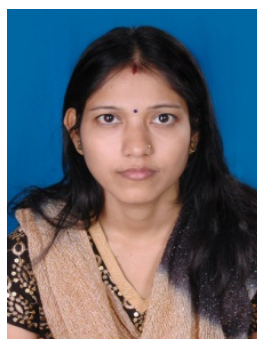

Smita Parija is presently continuing as research scholar in National Institute of Technology, Rourkela in Department of Electrical Engineering She received her Master degree from N.I.T Rourkela. Her specialization is in Communication System. Several publications in International journals and reputed international conference. She has supervised more than five PG Thesis and handling $\mathrm{B}$ tech projects also. Her research interests are wireless communication, neural network and Fuzzy logic.

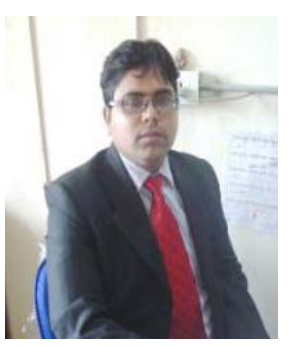

Dr. Santosh Kumar Nanda served as Director (Center of Research, Development and Consultancy) of Eastern Academy of Science \& Technology (EAST). Completed his PhD from National Institute of Technology, Rourkela. His research interests are Soft Computing, Artificial Intelligence, Image Processing, Prediction of machinery noise and vibration, Noise and vibration control, Mathematical modelling, Pattern Recognition. He has more than 40 research articles in reputed International Journals and International conferences etc. He is now Editor in Chief of International Journal of Logic and Computation (IJLP, CSC Journal Press, and Malaysia). He is now Editor of International Journal of Computer Application (IJCA), International Journal of Advancements in Computing Technology (IJACT), International Journal of Computer Applications (IJCA, Acta Press). International Journal of Open Problems in Computer Science and Mathematics (IJOPCM). Recently his name was selected for Editor in Chief for Asian Journal of Information Management, USA. In addition to this, his name was also selected for Regional Editor of International Journal of Applied 
Science, Regional Editor of International Journal of Artificial Intelligence, Regional Editor of International Research Journal of Information Technology and Regional Editor of Trends in Applied Science Research. Recently $\mathrm{He}$ is selected as Associate Editor of International Journal of Intelligent Systems and Applications, Hong Kong. He is now associate editor of International Journal of Intelligent System, Hong Kong. $\mathrm{He}$ is now editor of prestigious journal International Journal of Engineering and Manufacturing (IJEM), Hongkong.

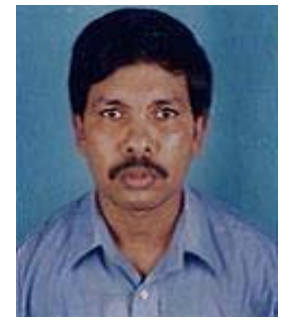

Dr. Prasanna Kumar Sahu received B.Sc Engineering (El\&TCE) and M.Sc Engineering (ESC) from Sambalpur University.Received his Ph. D degree from Jadavpur University, Kolkata in the year 2009. Currently he is Associate Professor in Department of Electrical Engineering at National Institute of Technology,Rourkelas.His research interest includes Micro and Nano Electronic Devices, VLSI and Communication Systems. He is also a life member of IEEE, IE and IETE.

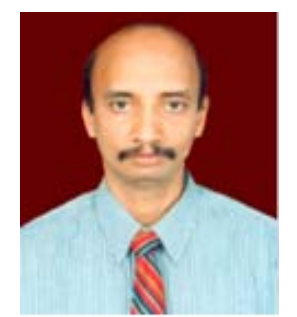

Dr. Sudhansu Sekhar Singh is working as Associate Professor in School of Electronics Engineering, KIITUniversity, Bhubaneswar ODISHA,INDIA.21 years of working experience out of which more than 14 years in teaching in reputed engineering colleges and Universities.He has done his ph.d from Jadavpur University,Kolkata,India and M.E Electronics system and communication from REC,Roourkela. Several publications in International journals and reputed international conference also e proceedings are to his credit. He has supervised more than twelve PG Thesis and examined couple of doctoral dissertations. His broad research area is in wireless and mobile communication, Specifically multicarrier CDMA,MIMOOFDM and Wireless Sensor Networks. 